\title{
Overview of ANTARES neutrino telescope: multimessenger results
}

Maurizio Spurio*, on behalf of the ANTARES Collaboration

INFN e Università degli Studi di Bologna

E-mail: spurio@bo.infn.it

Multimessenger astrophysics, connecting traditional astronomy with charged cosmic ray, $\gamma$-ray, gravitational wave and neutrino observations, has been made possible by the availability of experimental techniques developed, in most cases, for particle physics detectors. ANTARES is a large neutrino telescope located at $2475 \mathrm{~m}$ below the Mediterranean Sea level, $40 \mathrm{~km}$ offshore from Toulon (France). Data-taking started with the first 5 lines installed in 2007 and the full detector, completed in May 2008, will continue collecting data until 2020. The main objective of the telescope is to search for steady neutrinos sources and neutrinos from transient phenomena. For this reason, the Collaboration is involved in a wide multimessenger program to exploit the connection between neutrinos and other cosmic messengers: electromagnetic radiation from radio and visible to $\mathrm{X}$ - and $\gamma$-rays; charged cosmic rays; gravitational waves. Among all the possible astrophysical sources, transient sources increase the observation possibilities thanks to the suppression of atmospheric background in a well-defined space-time window.

In addition, thanks to the sub-degree angular resolution at high energy, the telescope has the possibility to significantly constrain models on the origin of part of the IceCube cosmic neutrinos in the Southern Sky, or on the blazar provenance of the highest-energy neutrinos. Furthermore, the combination of ANTARES and IceCube data allows to enhance the sensitivity for point-like neutrino sources in the Southern Hemisphere. Finally, ANTARES can also search for neutrinos produced by the annihilation of Dark Matter particles.

The recent ANTARES results are summarized in the following.

Neutrino Oscillation Workshop (NOW2018)

9 - 16 September, 2018

Rosa Marina (Ostuni, Brindisi, Italy)

* Speaker. 


\section{Physics objectives of the ANTARES telescope}

The search for astrophysical neutrinos in the TeV-PeV energy range is among the primary goals of neutrino telescopes. After the discovery of a cosmic neutrino diffuse flux by IceCube [1], the understanding of its origin has become a key mission in high-energy astrophysics. The ANTARES detector makes a valuable contribution for sources located in the Southern Sky, thanks to its excellent angular resolution in both the muon channel $\left(v_{\mu} \mathrm{CC}\right.$ interactions, yielding track-like events) and the cascade channel (shower-like events, due to $v_{e} \mathrm{CC}$ and by all neutrino flavors in $\mathrm{NC}$ interactions).

Point-sources searches. Searches for cosmic neutrino sources assuming a $E^{-2}$ spectrum using track- and shower-like events collected between 2007 and the end of 2015 have been recently reported [2]. After selection cuts, the sample consisted of 7622 track events and 180 shower events. Four different searches have been performed: a scan over the whole ANTARES visible sky; an investigation of 106 pre-selected astrophysical candidates; a dedicated analysis of the Galactic Center (GC) region; and a study of Sagittarius A* investigated as a possible extended source. No significant evidence of a cosmic neutrino signal over the atmospheric background has been found. Nevertheless, these searches provide the most sensitive limits for a large fraction of the Southern Sky (Fig. 1 top), especially in the energy range relevant for Galactic sources $\left(E_{v}<100 \mathrm{TeV}\right)$.

All-sky diffuse flux. A search for an all-flavor diffuse neutrino has been performed using 9 years of data [3]. The search includes both track-like and cascade-like events and concentrates on events coming from below the horizon to overcome the large background of down-going atmospheric muons. After energy-related selection cuts, 33 events are observed, with $24 \pm 7$ being expected from pure background and $\sim 8$ from the astrophysical flux. Even though the hypothesis of a null cosmic flux is not excluded with a large significance $(1.6 \sigma)$, the ANTARES result supports the intensity and spectral index on the cosmic neutrino flux observed by IceCube.

Searches from the Galactic Plane. A neutrino production concentrated in the Galactic plane is expected from cosmic ray (CR) interactions with Galactic gas and radiation fields. A search using ten years of ANTARES track and shower data, as well as seven years of IceCube track data have recently been finalized. The data are combined into a joint likelihood test for neutrino emission according to the recent models of Galactic CR diffusion. No significant excess has been found and the obtained limits start constraining the model parameter space [4].

Searches from Dark Matter. Neutrinos, as final product of a large variety of decay processes, are a good probe for an indirect search for dark matter (DM) in the form of Weakly Interacting Massive Particles (WIMPs). WIMPs are expected to accumulate in celestial objects (as the Sun, the Centre of the Earth, the GC) due to scattering with ordinary matter and the gravitation pull of these objects. Here, they can annihilate in pairs and subsequently produce standard model particles. ANTARES has performed searches for neutrinos from the direction of different massive objects. For instance, from the direction of the Sun the limits for the spin-dependent WIMP-nucleon crosssection improve upon those of current direct-detection experiments [5]. Then, 90\% C.L. limits on the thermally averaged annihilation cross-section, $\langle\sigma \mathrm{v}\rangle$, as a function of the WIMP mass and for different annihilation channels have been set using observation of the neutrino flux from the direction of the GC [6]. Currently active neutrino telescopes produce the most stringent limits in 

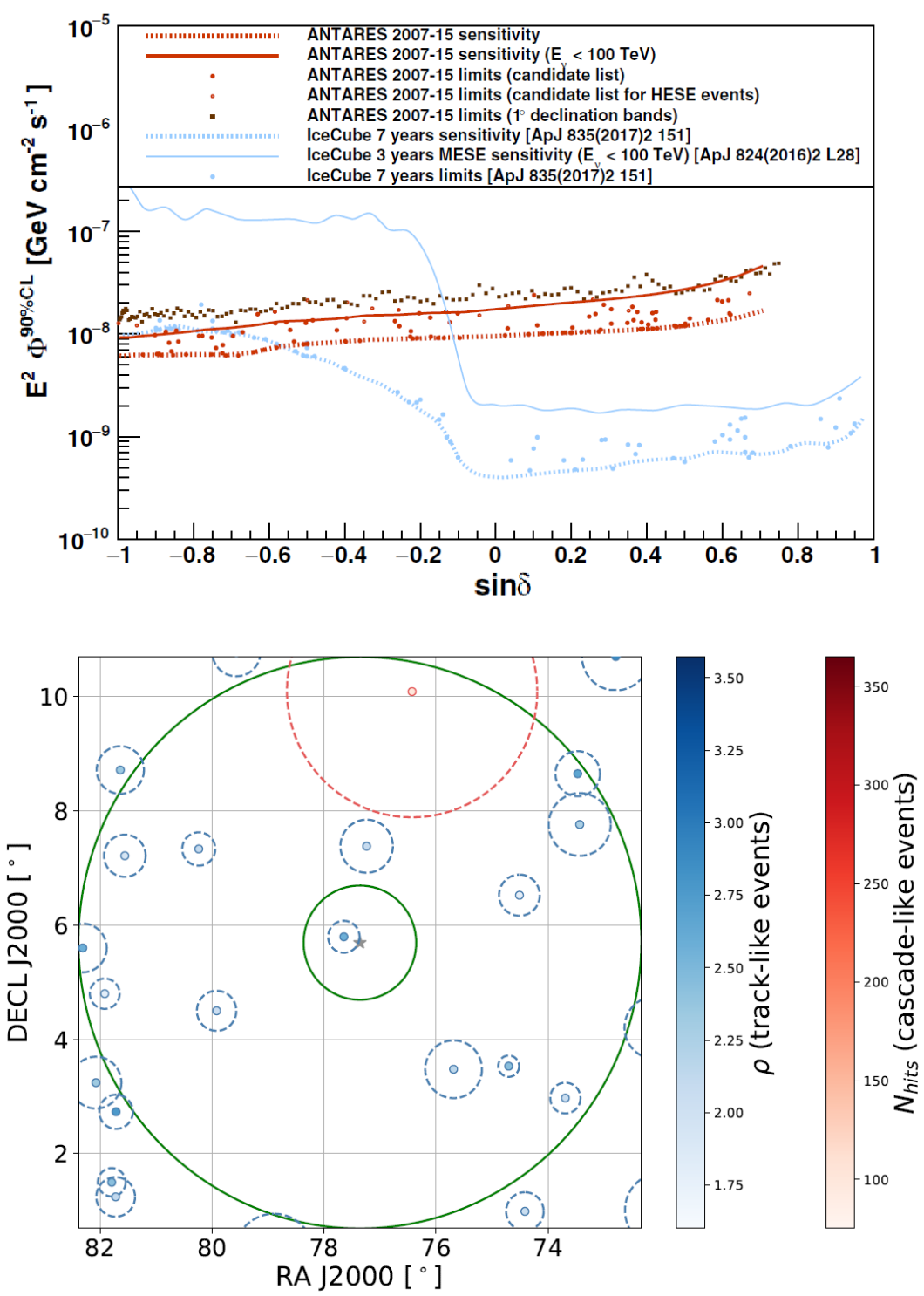

Figure 1: Top: Sensitivities (lines) and upper limits at 90\% C.L. (red circles) on the flux of one neutrino flavor from the investigated candidates assuming a $E^{-2}$ spectrum [2]. Bottom: Distribution of ANTARES events in the (RA, $\delta$ ) coordinates around the position of TXS 0506+056 [14].

the $M_{W I M P}>200 \mathrm{GeV} / \mathrm{c}^{2}$ range, surpassing even the direct-detection experiments.

\section{ANTARES Multimessenger searches}

Real-time follow-up analyses. ANTARES is able to handle external alerts rised by other experiments, distributed either through the Gamma-ray Coordinated Network (GCN) or under private agreements. Among them, the searches for neutrino counterparts of gravitational wave (GW) events detected by the LIGO and Virgo interferometers. ANTARES participated, together with IceCube, to a follow-up of the GW150914 event, providing the first constraint on high-energy neutrino 
emission from a binary black hole coalescence [7]. Neutrino telescopes are receiving GW alerts in real time since the beginning of $\mathrm{O} 2$ run. In this context, prompt searches for a neutrino counterpart to GW170817 - the first coalescence of two neutron stars- were performed [8]. No neutrinos directionally coincident with the source were detected within $\pm 500 \mathrm{~s}$ around the merger time. The non-detection is consistent with scenarios where a jet of highly energetic particles were produced off the line of sight of the source. Other real-time follow-up analyses have been performed after the detection transient events as GRBs and Fast Radio Bursts [9].

Alert triggering. Besides receiving alerts from other observatories, ANTARES also triggers follow-up of neutrinos of potential astrophysical origin [10]. Thanks to the very short alertgeneration time (a few seconds) and half-sky simultaneous coverage, ANTARES is well-suited to detect transient sources. In this case, an alert message is generated to trigger robotic optical telescopes (TAROT, MASTER, ZADKO), radio telescopes (MWA), X-ray satellites (Swift-XRT, INTEGRAL), and ground-based $\gamma$-ray observatories (H.E.S.S., HAWC). The criteria for triggering specific instruments depend on the desired false alarm rate. General properties of the triggers are either high energetic events, doublets in a time window of 15 minutes or events of which the origin is compatible with a known close-by galaxy. Since 2009, ANTARES has sent more than 300 alerts.

Off-line analyses. Off-line analyses are performed to search for neutrino counterparts to catalogued flaring sources as galactic X-ray binaries [11], microquasars, blazars and GRBs [12]. These objects are expected to share the same physical mechanisms based on the accretion of gas onto a black hole which power the relativistic jets of material ejected from both sides of the compact object. No cosmic neutrino in correlation with these events has been detected, allowing some of the more optimistic models for hadronic acceleration in these sources to be rejected at 90\% C.L. Finally, a study to constrain a possible origin of the IceCube astrophysical signal from transient sources searching for additional neutrinos is currently in progress.

Search for Neutrinos from TXS 0506+056. A recent HE neutrino detected by the IceCube experiment (IC-170922A) was connected with observations in $\gamma$-rays and at larger wavelengths from blazar TXS 0506+056 [13]. This may indicate that this blazar (a BL Lac object, at redshift $z=0.3365 \pm 0.0010)$ is an individually identifiable source of HE neutrinos. Two independent analyses on archival IceCube neutrino events at the position of the blazar confirmed an excess at the level of 3.5 $\sigma$. ANTARES has searched for neutrino candidates, associated with the IC-170922A event or from the direction of TXS 0506+056, using three strategies. The first study refers to the prompt follow-up of the IceCube alert, which yields a null result. The second search is based on the standard time-integrated method employed in [2] to search for point-like neutrino sources. This time-integrated study (covering a data period from 2007 to 2017) fits 1.03 signal events, which corresponds to a p-value of $3.4 \%$ (not considering trial factors). One track-like event mostly influences the fit (see Fig. 1 bottom) whose position is within $1 \sigma$ from the source position. This event occurred on 2013, December 12. Only for two other astrophysical objects in our candidate list [2], a smaller p-value had been found. When considering that 107 sources have been investigated, the post-trial p-value for TXS $0506+056$ corresponds to $87 \%$. Finally, the third strategy uses the information from the IceCube time-dependent analysis reporting a bursting activity centered on December 13, 2014, as input for an ANTARES time-dependent analysis. No correlated events are found. 


\section{References}

[1] M. G. Aartsen et al., Science 342 (2013) 1242856.

[2] A. Albert et al., Phys. Rev. D96 (2017) 082001; arXiv:1706.01857

[3] A. Albert et al., ApJ 853 (2018) L7; arXiv:1711.07212

[4] A. Albert et al., arXiv:1810.10693.

[5] S. Adrián-Martínez et al., Phys.Lett. B759 (2016) 69; arXiv:1603.02228

[6] A. Albert et al., Phys.Lett. B769 (2017) 249; arXiv:1612.04595

[7] S. Adrián-Martínez et al., Phys. Rev. D93 (2016) 122010; arXiv:1602.05411

[8] A. Albert et al., ApJL 850 (2017) L35; arXiv:1710.05839

[9] S. Bhandari et al., MNRAS 475 (2018) 1427; arXiv:1711.08110

[10] S. Adrián-Martínez et al., JCAP 1602 (2016) 062; arXiv:1508.01180

[11] A. Albert et al., JCAP 1704 (2017) 019; arXiv:1609.07372

[12] A. Albert et al., MNRAS 469 (2017) 906 ; arXiv:1612.08589

[13] IceCube et al., Science 361 (2018); arXiv:1807.08816

[14] A. Albert et al., ApJL 863 (2018) L30; arXiv:1807.04309 